\title{
Simulation of S- and L-Band FSS Reflector Antenna
}

\author{
G. Catton, H. G. Espinosa, A. Dewani, S. G. O’Keefe \\ Griffith University, Brisbane, Australia \\ g.catton@griffith.edu.au
}

\begin{abstract}
A dual-band FSS (Frequency Selective Surface) reflector antenna that operates in the S- and L-bands with gains of $8 \mathrm{dBi}$ and $11 \mathrm{dBi}$ at $1 \mathrm{GHz}$ and $2.5 \mathrm{GHz}$ respectively is demonstrated. The antenna has been simulated successfully showing good results in CST MWS ${ }^{\circledR}$ (Microwave Studio) EM (Electromagnetic) simulation software. The reflector antenna consists of a dual-band trap dipole antenna situated over an FSS upper parabolic reflector and a PEC (Perfect Electrical Conductor) lower parabolic reflector. The convoluted design of the FSS gives it angular and polarisation stability with a stopband centred at $2.5 \mathrm{GHz}$.
\end{abstract}

Keywords-Convoluted; dual-band; FSS (Frequency Selective Surface); PEC (Perfect Electrical Conductor); sub-reflector.

\section{INTRODUCTION}

It is well known that the use of a parabolic reflecting surface to focus the transmission of EM (Electromagnetic) waves from conventional horn, dipole and other antennas will increase antenna gain. In 1897 Marconi first used a reflector to increase his antenna's gain [1] and in 1919 Marconi and Franklin patented a reflector antenna that used only parabolically curved metal strips or rods instead of the solid faced parabolic reflector Marconi had used previously. They found that when using only three half-wavelength rods, equally spaced, in parallel, correctly polarised, and positioned at the correct focal length from the antenna, "the range may be increased from a $00 \%$ to $500 \%$ as against $80 \%$ obtained with the simple reflectors" [2]. Marconi and Franklin's curved rod reflectors are similar to the classic FSS unit cell designs used as FSS sub-reflectors today.

As its name implies, an FSS (Frequency Selective Surface) will behave differently depending on the frequency of an applied EM wave. A band-pass FSS will allow a signal at a certain frequency to pass through with minimal attenuation while reflecting other frequencies [3]. This characteristic makes the FSS particularly useful when it is used as a subreflector in antenna reflector systems. The use of FSS subreflectors allows, for example, feed antennas in separate locations to use a single main reflector thus decreasing size and cost of the antenna and providing the convenience of not needing to realign the reflector dish for each different frequency band. Such an FSS sub-reflector system was used on the Voyager and Galileo spacecraft to reflect X-band while Sband passed straight through thus allowing for X- and S-Band communication from the same main reflector dish [4]. The Cassini space mission used a tri-band FSS reflector system that employed an initial FSS to reflect X-band while passing through S- and Ku-bands and then a secondary FSS that reflected Ka-band and allowed S-, X- and Ku-bands to pass through it [5].

The frequency response of a an FSS is also sensitive to the angle of incidence and polarisation of an applied EM wave but certain FSS unit cells designs make them less sensitive in that regard. Previous research has found that an FSS will be less sensitive to the angle of incidence and polarisation of an EM wave when its unit cell is small when compared to the wavelength of the FSS resonant frequency [6]. In practise this means that at frequencies in the X-Band or higher, it is possible to design FSS unit cells small enough to achieve robustness to angular and polarisation variations of the applied EM field using simple element designs, such as rings or squares. However, at frequencies in the C-band and lower it becomes difficult to fabricate a unit cell that is small enough to ensure the FSS is adequately insensitive to the angular and polarisation variations of the applied EM field. Recent research [7] has found that by convoluting the unit cell design it is possible to achieve resonant frequencies in the C-Band or lower and still retain robustness to angle of incidence and polarisation variations. FSS with reject frequencies in the Sand L-bands have been developed at Griffith University by screen printing a suitably convoluted unit cell onto a thin flexible substrate [8]. The flexibility of this FSS substrate makes it suitable to curve into a parabolic reflector and use as the upper reflector for this antenna.

\section{FSS REFLECTOR ANTENNA DESIGN}

The reflector antenna, which is shown in Fig. 1, was designed and simulated in CST MWS ${ }^{\circledR}$ (www.cst.com). It consists of a $1 \mathrm{GHz}$ half-wave dipole antenna with two 2.5 $\mathrm{GHz}$ parallel LC resonator traps located approximately halfway along each element of the dipole. The values of the inductor and capacitor are $\mathrm{L}=2.7 \mathrm{nH}$ and $\mathrm{C}=1.5 \mathrm{pF}$ respectively and cause a resonance at $2.5 \mathrm{GHz}$. This resonance causes the trap to appear as an open circuit at $2.5 \mathrm{GHz}$ which effectively cuts the dipole in half and creates a half-wavelength dipole at that frequency. The traps cause the dipole to have two bands which are centred at $1 \mathrm{GHz}$ and $2.5 \mathrm{GHz}$. There are two parabolic reflectors located at one quarter wavelength for each band from the trap dipole. The upper parabolic reflector is an FSS which has a stop-band centred at $\mathrm{f}_{\text {stop }}=2.5 \mathrm{GHz}$ so it is very reflective at that frequency while other frequencies pass through with minimal attenuation. The lower parabolic reflector is a PEC (Perfect Electrical Conductor) and will reflect all frequencies but as it is located at one quarter wavelength at $1 \mathrm{GHz}$ from the dipole, the $1 \mathrm{GHz}$ EM waves returning to the dipole will be in phase and thus add constructively to those leaving the dipole in the $\mathrm{Z}$ direction. The $2.5 \mathrm{GHz}$ EM waves reflected by the FSS 
will also be in phase and add constructively with the $2.5 \mathrm{GHz}$ waves leaving the dipole in the $\mathrm{Z}$ direction. The additive effects of the $1 \mathrm{GHz}$ and $2.5 \mathrm{GHz}$, in-phase, EM waves returning to the dipole has the effect of increasing the gain at those frequencies.

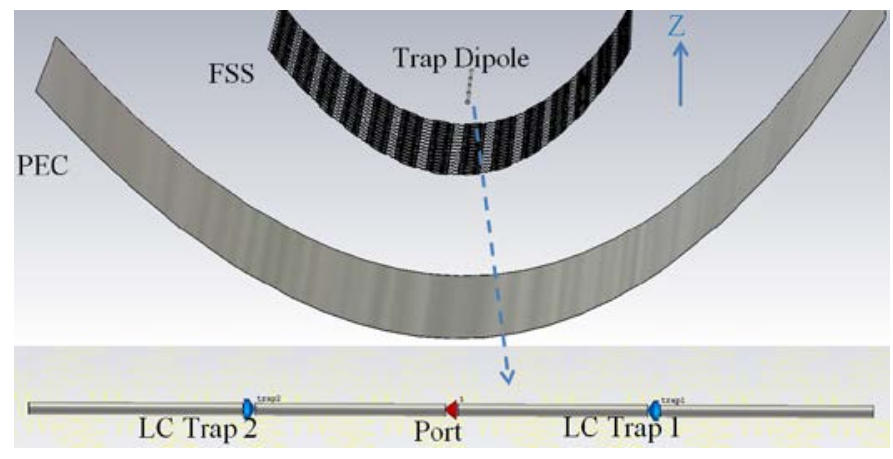

Fig. 1. Reflector antenna design in CST MWS ${ }^{\circledR}$ with bottom inset showing an enlarged view of the trap dipole antenna.

\section{RESULTS}

\section{A. Reflection Coefficient and Efficiency}

As shown from the CST MWS ${ }^{\circledR}$ results in Fig. 2, the antenna resonates at $1 \mathrm{GHz}$ with a voltage reflection coefficient of $\mathrm{S}_{11}=-35 \mathrm{~dB}$ and also at $2.5 \mathrm{GHz}$ with a reflection coefficient of $\mathrm{S}_{11}=-25 \mathrm{~dB}$. The $-10 \mathrm{~dB}$ bandwidth for $1 \mathrm{GHz}$ is $250 \mathrm{MHz}$ and for $2.5 \mathrm{GHz}$ it is $190 \mathrm{MHz}$. The radiation efficiencies at $1 \mathrm{GHz}$ and $2.5 \mathrm{GHz}$ are both close to $0 \mathrm{~dB}$ so the antenna is well tuned for the frequencies of operation.

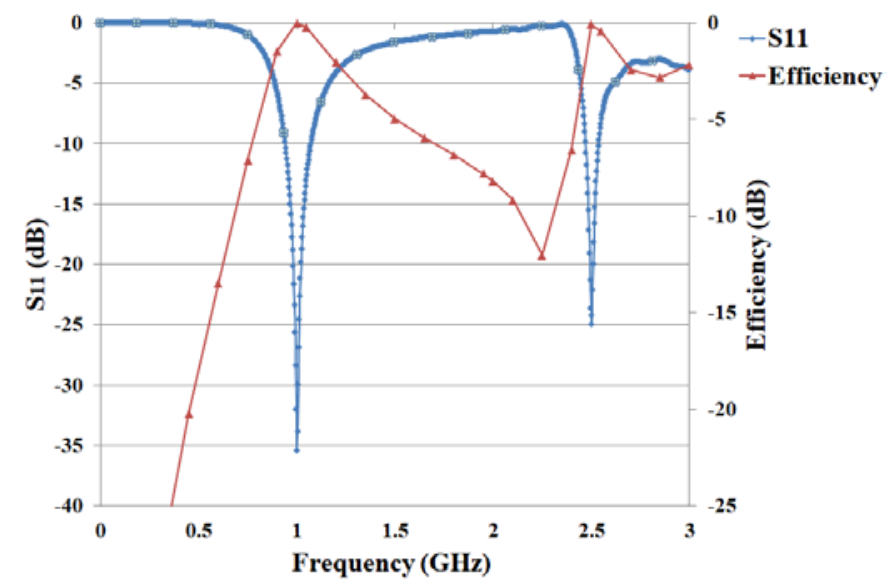

Fig. 2. $\mathrm{S}_{11}$ reflection coefficient and radiation efficiency of the dual-band reflector antenna simulated in CST MWS ${ }^{\circledR}$.

\section{B. Gain}

Fig. 3. shows the gain for the reflector antenna is $8 \mathrm{dBi}$ and $11 \mathrm{dBi}$ at $1 \mathrm{GHz}$ and $2.5 \mathrm{GHz}$ respectively at $\theta=0^{\circ}, \varphi=0^{\circ}$ and $\theta=0^{\circ}, \varphi=90^{\circ}$. The HPBW (Half Power Beamwidth) at $1 \mathrm{GHz}$ is $70^{\circ}$ at $\varphi=0^{\circ}$ and $50^{\circ}$ at $\varphi=90^{\circ}$. The HPBW at $2.5 \mathrm{GHz}$ is $46^{\circ}$ at $\varphi=0^{\circ}$ and $30^{\circ}$ at $\varphi=90^{\circ}$.

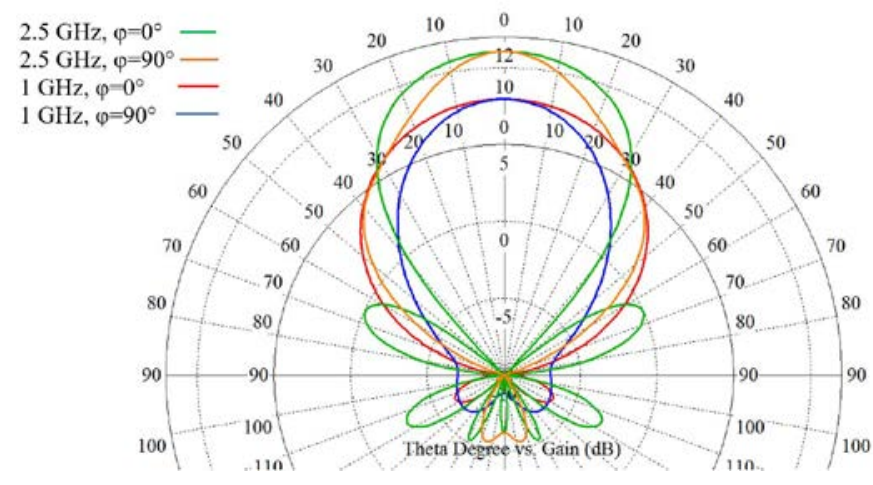

Fig. 3. Polar plot of the reflector antenna gain at different angles of $\theta$ for $\varphi=$ $0^{\circ}$ and $\varphi=90^{\circ}$ at $1 \mathrm{Ghz}$ and $2.5 \mathrm{Ghz}$ as simulated in CST MWS ${ }^{\circledR}$.

\section{DISCUSSION}

The FSS used in this reflector antenna has enabled dualband operation at $1 \mathrm{GHz}$ and $2.5 \mathrm{GHz}$. The convoluted design of the FSS gives it a rejection frequency at $2.5 \mathrm{GHz}$ allowing it to be used as a sub-reflector at frequencies lower than previous FSS sub-reflector designs. The reflector antenna has good gain at each band. The gain at $1 \mathrm{GHz}$ is $8 \mathrm{dBi}$ which is $5.85 \mathrm{dBi}$ greater than that of a simple half-wave dipole on its own $(2.15$ $\mathrm{dBi}$ [9]). And the gain at $2.5 \mathrm{GHz}$ is $11 \mathrm{dBi}$ which is $8.85 \mathrm{dBi}$ greater than a half-wave dipole. The frequencies of this reflector antenna means it has uses in applications such as WiFi, LTE (Long Term Evolution), mobile phone communication, and other S- and L-Band applications. It is planned to build a prototype of this reflector antenna, carry out field measurements and compare them to simulated results.

\section{REFERENCES}

[1] G. Marconi, "Improvements in transmitting electrical impulses and signals, and in apparatus therefor,” Brit. Patent No.12,039, July 2, 1897.

[2] G. Marconi anmd C.S. Franklin, "Refelector for use in wireless telegraphy and telephony,” U.S.Patent 1301473, April 22, 1919.

[3] B.A. Munk, Frequency Selective Surfaces: Theory and Design, Wiley, New York, 2000.

[4] Y. Rahmat-Samii and M. Gatti, "Far-field patterns of spaceborne antennas from plane-polar near-field measurements," IEEE Trans. Antennas Propagat. vol. 33, pp. 638-648, June 1985.

[5] J. Huang, T. Wu, and S. Lee, "Tri-band frequency selective surface with circular ring elements,” IEEE Trans. Antennas Propagat. vol. 32, no. 2, pp. 166-175, Feb. 1994.

[6] K. Sarabandi and N. Behdad, “A frequency selective surface with miniaturized elements,” IEEE Trans. Antennas and Propagat. vol. 55, no. 5, pp. 1239-1245, May 2007.

[7] E.A. Parker and A.N.A. El Sheikh, "Convoluted dipole array elements," IEEE Electron. Lett. vol. 27, no. 4, pp. 322-323, Feb. 1991.

[8] A.A. Dewani, S.G. O’Keefe, D.V. Thiel, and A. Galehdar, "Miniaturised meandered square frequency selective surface on a thin dielectric with a selective transmission,” Flex. Print. Electron, vol. 1, no.2, 025001, April 2016.

[9] S.J. Orfanidis, "Linear and Loop Antennas," in Electromagnetic Waves and Antennas, 2002, ch. 15, p. 502. [Online]. Available: http://eceweb1.rutgers.edu/ orfanidi/ewa 